OPEN ACCESS

Edited by:

Catherine Ropert

Federal University of Minas

Gerais, Brazil

Reviewed by:

Tarek A. Ahmad,

Bibliotheca Alexandrina, Egypt

Duolao Wang,

Liverpool School of Tropical Medicine

United Kingdom

${ }^{*}$ Correspondence:

Yufeng Chu

chunancy@163.com

Mei Meng

mmB1273@rjhn.com.cn

tThese authors have contributed equally to this work

Specialty section:

This article was submitted to Infectious Diseases - Surveillance,

Prevention and Treatment,

a section of the journal

Frontiers in Medicine

Received: 16 September 2020 Accepted: 25 January 2021

Published: 12 February 2021

Citation:

Sha J, Qie G, Yao Q, Sun W, Wang C,

Zhang $Z$, Wang $X$, Wang $P$, Jiang J,

Bai X, Chu Y and Meng M (2021) SeX Differences on Clinical Characteristics,

Severity, and Mortality in Adult

Patients With COVID-19: A

Multicentre Retrospective Study.

Front. Med. 8:607059.

doi: 10.3389/fmed.2021.607059

\title{
Sex Differences on Clinical Characteristics, Severity, and Mortality in Adult Patients With COVID-19: A Multicentre Retrospective Study
}

\section{Jing Sha ${ }^{1 \dagger}$, Guoqiang Qie ${ }^{1 \dagger}$, Qingchun Yao ${ }^{1}$, Wenqing Sun ${ }^{2}$, Cuiyan Wang ${ }^{3}$, Zhongfa Zhang ${ }^{4}$, Xingguang Wang ${ }^{5}$, Peng Wang ${ }^{1}$, Jinjiao Jiang ${ }^{1}$, Xue Bai ${ }^{1}$, Yufeng Chu ${ }^{1 *}$ and Mei Meng ${ }^{6 *}$}

${ }^{1}$ Department of Critical Care Medicine, Shandong Provincial Hospital Affiliated to Shandong First Medical University, Shandong Provincial Hospital Affiliated to Shandong University, Shandong University, Jinan, China, ${ }^{2}$ Department of Intensive Care Unit, Shandong Provincial Chest Hospital, Jinan, China, ${ }^{3}$ Shandong Medical Imaging Research Institute Affiliated to Shandong University, Jinan, China, ${ }^{4}$ Jinan Infectious Diseases Hospital, Shandong University, Jinan, China, ${ }^{5}$ Department of Pulmonary and Critical Care Medicine, Shandong Provincial Hospital Affiliated to Shandong First Medical University, Jinan, China, ${ }^{6}$ Department of Critical Care Medicine, Ruijin Hospital, Ruijin Hospital North, Shanghai Jiao Tong University School of Medicine, Shanghai, China

Background: Coronavirus disease-2019 (COVID-19) epidemic is spreading globally. Sex differences in the severity and mortality of COVID-19 emerged. This study aims to describe the impact of sex on outcomes in COVOD-19 with a special focus on the effect of estrogen.

Methods: We performed a retrospective cohort study which included 413 patients (230 males and 183 females) with COVID-19 from three designated hospitals in China with a follow up time from January 31, 2020, to April 17, 2020. Women over 55 were considered as postmenopausal patients according to the previous epidemiological data from China. The interaction between age and sex on in-hospital mortality was determined through Cox regression analysis. In addition, multivariate Cox regression models were performed to explore risk factors associated with in-hospital mortality of COVID-19.

Results: Age and sex had significant interaction for the in-hospital mortality $(P<0.001)$. Multivariate Cox regression showed that age $(\mathrm{HR} 1.041,95 \% \mathrm{Cl} 1.009-1.073$, $P=0.012)$, male sex ( $\mathrm{HR} 2.033,95 \% \mathrm{Cl} 1.007-2.098, P=0.010)$, the interaction between age and sex (HR 1.118, 95\% Cl 1.003-1.232, $P=0.018)$, and comorbidities (HR 9.845, 95\% Cl 2.280-42.520, $P=0.002)$ were independently associated with in-hospital mortality of COVID-19 patients. In this multicentre study, female experienced a lower fatality for COVID-19 than male (4.4 vs. 10.0\%, $P=0.031)$. Interestingly, stratification by age group revealed no difference in-hospital mortality was noted in women under 55 compared with women over 55 (3.8 vs. $5.2 \%, P=0.144$ ), as well as in women under 55 compared with the same age men (3.8 vs. 4.0\%, $P=0.918$ ). However, there was significantly difference in women over 55 with men of the same age group (5.2 vs. $21.0 \%, P=0.007$ ). Compared with male patients, female patients 
had higher lymphocyte $(P<0.001)$ and high-density lipoprotein $(P<0.001)$, lower high sensitive $\mathrm{c}$ reaction protein level $(P<0.001)$, and lower incidence rate of acute cardiac injury (6.6 vs. $13.5 \%, P=0.022$ ).

Conclusion: Male sex is an independent risk factor for COVID-19 in-hospital mortality. Although female mortality in COVID-19 is lower than male, it might not be directly related to the effect of estrogen. Further study is warranted to identify the sex difference in COVID-19 and mechanisms involved.

Keywords: COVID-19, sex, estrogen, menopause, mortality, China

\section{INTRODUCTION}

The whole world is currently under the effect of the ongoing epidemic of coronavirus disease-2019 (COVID-19), caused by a novel coronavirus termed severe acute respiratory syndrome coronavirus (SARS-CoV-2). As of 17 June 2020, the World Health Organization (WHO) has reported 8,061,520 confirmed cases and 440,290 deaths in 216 countries and regions, and COVID-19 has become a public health emergency of international concern (PHEIC) (1). Although most of the COVID-19 patients are non-severe and self-limited, there are still $16 \%$ severe cases and $3.1 \%$ died in China $(2-5)$.

The current studies showed that advanced age and comorbidities were closely related to worse prognosis (6-9). Recently a retrospective multicentre cohort study demonstrated older COVID-19 patients tended to have relatively more severe clinical infections and poorer clinical outcomes associated with COVID-19 compared with younger patients in Jiangsu of China (10). In addition, in a multicentre Italian CORIST study including 3,894 patients with SARS-CoV-2 infection found advanced age at hospital admission was one of powerful predictors of higher in-hospital death (11). Meanwhile, evidence of sex differences in COVID-19 severity emerged, where the morbidity and mortality were all higher among males than females (12-14). A male bias in COVID-19 mortality was reported in 37 of the 38 countries that have provided sexdisaggregated data. Scully et al. showed that the average male case fatality rate (CFR) across 38 countries was 1.7 times higher than the average female CFR (15). Previous epidemiological studies showed the proportion of individuals infected and CFRs in severe acute respiratory syndrome (SARS)-CoV and Middle East respiratory syndrome (MERS)-CoV were higher in males than that of females $(16,17)$. The causes of Sex differences following virus infections are multifactorial, including differences in steroid hormones, immune response $\mathrm{X}$-linked genes, disease-susceptibility genes in sexes, and gender-related social factors (18-20).

Studies have linked increased susceptibility to infection with circulating steroid hormone concentrations $(18,19,21)$. Traditionally, sex steroid hormones, especially the estrogen in females, have been considered for their immunomodulatory properties. Animal study indicated that ovariectomy or treating female mice with estrogen receptor antagonist increased mortality, indicating a protective effect for estrogen receptor signaling in mice infected with SARS-CoV (22). Thus, the lower incidence of severe COVID-19 in female patients might be related to the protective effect of estrogen. However, as yet, few studies have focused on sex differences in clinical characteristics and laboratory tests of COVID-19, especially whether estrogen affects the occurrence and development of COVID-19. Therefore, this study aims to analysis the sex differences on clinical characteristics, severity and mortality in adult patients with COVID-19, and explore possible mechanisms, with a special focus on premenopausal and postmenopausal women.

\section{MATERIALS AND METHODS}

\section{Study Design and Participants}

The multicentre retrospective cohort study was conducted at three hospitals designated for the treatment of COVID-19, including Jinan Infectious diseases Hospital in Shandong, Shandong Provincial Chest Hospital in Shandong, and Huanggang Central Hospital in Hubei. The recruitment period was from January 31, 2020, to April 17, 2020. The diagnosis of COVID-19 was made based on the National Health Commission of China guidance (23). The presence of SARSCOV-2 in respiratory specimens was confirmed using real-time reverse-transcriptase polymerase chain reaction (RT-PCR) assay were performed in accordance with the protocol described previously (2). The patients that are pregnant or $<18$ years old were excluded. As of April 17, 2020, all included patients were discharged or died. In addition, the vast majority of city women were postmenopausal by age $55(24,25)$, according to the previous epidemiological data from China, which was consistent with our study. Thus, patients were divided into two groups according to the age of 55 to explore the role of estrogen in the progression of COVID-19. The study was approved by the institutional review board of Jinan Infectious diseases Hospital, Shandong Provincial Chest Hospital, and Huanggang Central Hospital.

\section{Data Collection}

Two physicians reviewed clinical electronic medical records and laboratory findings for all patients with SARS-CoV-2 infection, and then a third researcher determined any differences between interpretations of the two primary reviewers. The demographic data, menstrual history of women, clinical characteristics and laboratory results were collected at admission. We also evaluated and gathered complications, treatment and clinical outcomes 
(discharged alive or dead) at the end of study, by using a standardized case-report form. For patients with a readmission during the study period, data from the first admission were presented. Sequential Organ Failure Assessment (SOFA) scores were calculated using the worst value of physiological variables within 24 h of presentation.

\section{Diagnostic and Grading Criteria for COVID-19}

The disease severity of COVID-19 patients was divided into severe and non-severe conditions, defined according to the American Thoracic Society guidelines for community-acquired pneumonia (26). Severe COVID-19 should reach the following either one major criterion or three or more minor criteria. In detail, Minor criteria included respiratory rate more than 30 breaths per minute, $\mathrm{PaO} 2 / \mathrm{FIO} 2$ ratio lower than 250, multilobar infiltrates confusion or disorientation, blood urea nitrogen level more than $7.1 \mathrm{mmol} / \mathrm{L}$, white blood cell count $<4.0 \times 10^{9}$ per L, platelet count $<100 \times 10^{12}$ per L, core temperature lower than $36^{\circ} \mathrm{C}$, and hypotension requiring aggressive fluid resuscitation. Major criteria included septic shock with need for vasopressors, or mechanical ventilation. Fever was defined as axillary temperature of at least $37.3^{\circ} \mathrm{C}$. Septic shock was defined according to the 2016 Third International Consensus Definition for Sepsis and Septic Shock (27). Acute kidney injury was diagnosed according to the KDIGO clinical practice guidelines (28) and acute respiratory distress syndrome (ARDS) was diagnosed according to the Berlin Definition (29). Acute cardiac injury was diagnosed if serum levels of cardiac biomarkers (e.g., high-sensitive cardiac troponin I) were above the 99th percentile upper reference limit, or if new abnormalities were shown in electrocardiography and echocardiography (2).

\section{Outcomes}

The primary outcome was in-hospital mortality. The secondary outcomes were including disease severity, development of acute respiratory distress (ARDS), acute cardiac injury, acute liver injury, sepsis shock and acute kidney injury (AKI).

\section{Statistical Analysis}

Patients were divided into two groups according to sex, and the subgroup analysis was performed at the cut-off point of 55 years old according to the age of menopause in women. Female patients and male patients were grouped by age into the younger group(less than or 55 years old) and the older group (above 55 years old) for comparison. Continuous variables were expressed as mean \pm standard deviation (SD) or medians (interquartile range, IQR) values. Categorical data were summarized as frequency rates and percentages. The comparison between the two groups was conducted using $t$ tests or Mann-Whitney $U$ tests for continuous variables, and chi-squared tests or Fisher's exact tests for categorical variables. The interaction between age and sex on in-hospital mortality was determined through Cox regression analysis. In addition, multivariate Cox regression models were performed to explore risk factors associated with in-hospital mortality of COVID-19. Considering the total number of death cases $(n=31)$ in this study and to avoid overfitting in the model, four factors with significant association with mortality in univariate regression analyses (sex, age, the interaction between age and sex, and comorbidities) were chosen for multivariate analysis on the basis of previous findings and clinical constraints $(8,10,12,13,30)$. Hazard ratios (HRs) with $95 \%$ confidence intervals (CIs) and the corresponding $P$ values were calculated for each risk factor. Kaplan-Meier estimator was generated to estimate the survival curves and logrank test was used to compare the survival probability between male and female groups. $P<0.05$ was considered statistically significant. The variables that had $>5 \%$ of values missing were excluded. Simple data imputation was done for missing data $<5 \%$, using the median for skewed distribution data, or the mode for dichotomous data. All analyses were conducted with SPSS software, version 22.0 (SPSS Inc. Chicago, Illinois, United States).

\section{RESULTS}

\section{Basic Characteristics}

A total of 441 COVID-19 patients (range, 2-89 years) were hospitalized in the three designated hospital from Jan 31, 2020

TABLE 1 | Sex-specific demographic and clinical characteristics of COVID-19 patients.

\begin{tabular}{|c|c|c|c|}
\hline Variables & Male $(n=230)$ & Female $(n=183)$ & $P$-value \\
\hline Age, Median (IQR),y & $56(46,67)$ & $59(49,67)$ & 0.094 \\
\hline current or ever smoking & $18(7.8)$ & $0(0)$ & $<0.001$ \\
\hline Drinking & $17(7.4)$ & $0(0)$ & $<0.001$ \\
\hline \multicolumn{4}{|l|}{ Comorbidities } \\
\hline COPD & $6(2.6)$ & $1(0.5)$ & 0.139 \\
\hline DM & $30(13.0)$ & $24(13.1)$ & 0.983 \\
\hline Hypertension & $70(30.4)$ & $47(25.7)$ & 0.287 \\
\hline Heart disease & $15(6.5)$ & $11(6.0)$ & 0.832 \\
\hline Kidney disease & $6(2.6)$ & $4(2.2)$ & 0.781 \\
\hline Liver disease & $7(3.0)$ & $5(2.7)$ & 0.851 \\
\hline Shock & $8(3.5)$ & $6(3.3)$ & 0.911 \\
\hline Tumor & $6(2.6)$ & $7(3.8)$ & 0.482 \\
\hline Immune disease & $4(1.7)$ & $4(2.2)$ & 0.744 \\
\hline \multicolumn{4}{|l|}{ Symptoms } \\
\hline Fever & $208(90.4)$ & $150(82.0)$ & 0.012 \\
\hline Cough & $178(77.4)$ & $142(76.1)$ & 0.961 \\
\hline Expectoration & $81(35.2)$ & $57(31.1)$ & 0.384 \\
\hline Chest distress & $110(47.8)$ & $92(50.2)$ & 0.621 \\
\hline Chest pain & $5(2.2)$ & $8(4.4)$ & 0.204 \\
\hline Hemoptysis & $4(1.7)$ & $2(1.1)$ & 0.697 \\
\hline Headache & $12(5.2)$ & $11(6.0)$ & 0.727 \\
\hline Myalgia & $24(10.4)$ & $24(13.1)$ & 0.399 \\
\hline Fatigue & $83(36.1)$ & $71(38.8)$ & 0.571 \\
\hline Gastrointestinal & $33(14.3)$ & $35(19.1)$ & 0.193 \\
\hline SOFA Score, median (IQR) & $1(0,2)$ & $1(0,2)$ & 0.022 \\
\hline
\end{tabular}

Data are $n(\%)$ unless specified otherwise. $P<0.05$ was considered statistically significant. COVID-19, coronavirus disease 2019; IQR, interquartile range; COPD, chronic obstructive pulmonary disease; NS, no significance; DM, diabetes mellitus; SOFA, Sequential Organ Failure Assessment. Bold value means $P<0.05$. 
to Apr 17, 2020. After excluding one pregnant patient, 11 patients $<18$ years old, and 16 patients without available key information in their medical records, we included 413 patients in the final analysis, among whom 230 were males and 183 were females. Table 1 presented the sex-specific demographic and clinical characteristics of the all patients with COVID19. The median age of all cases was 58 years old (IQR 47-67 years), and it had not difference between males and females. Comorbidities were common for both sexes, but no significant difference. Regarding the symptoms, fever, cough and chest distress were the most common on admission among both men and women. However, a higher percentage of men had fever (90.4\% vs. $82.0 \%, P=0.012$ ). Additionally, SOFA score differed significantly between males and females.

\section{Laboratory Results}

Some laboratory results at admission showed significant differences between male and female patients $(P<0.05)$. Male cases had substantially increased hemoglobin, alanine amino transferase (ALT), creatinine, creatine kinase, creatine kinase isoenzyme-MB (CK-MB), and procalcitonin, while significantly

TABLE 2 | Sex-specific laboratory results of COVID-19 patients.

\begin{tabular}{|c|c|c|c|c|}
\hline Variables & $\begin{array}{l}\text { Normal } \\
\text { Range }\end{array}$ & $\begin{array}{c}\text { Male } \\
(n=230)\end{array}$ & $\begin{array}{c}\text { Female } \\
(n=183)\end{array}$ & $P$-value \\
\hline White blood cell $\left(\times 10^{9} / \mathrm{L}\right)$ & $3.5-9.5$ & $7.90(6.68,10.2)$ & $7.61(6.54,9.81)$ & 0.172 \\
\hline Neutrophil $\left(\times 10^{9} / \mathrm{L}\right)$ & $1.8-6.3$ & $6.13(4.90,8.37)$ & $5.98(4.67,8.55)$ & 0.434 \\
\hline Lymphocyte (×109/L) & $1.1-3.2$ & $1.23(1.0,1.62)$ & $1.36(1.11,1.72)$ & $<0.001$ \\
\hline Hemoglobin (g/L) & $316-354$ & $130(123,139)$ & $117(108,124)$ & $<0.001$ \\
\hline Platelet (×109/L) & $125-350$ & $251(220,313)$ & $265(233,313)$ & 0.030 \\
\hline D-dimer $(\mu \mathrm{g} / \mathrm{ml})$ & $0-1.5$ & $0.80(0.43,2.06)$ & $0.92(0.43,2.25)$ & 0.483 \\
\hline ALT (U/L) & $9-50$ & $36(22,53)$ & $22(15,35)$ & $<0.001$ \\
\hline LDH (U/L) & $120-250$ & $344(286,446)$ & $334(278,419)$ & 0.165 \\
\hline IL-6 (pg/ml) & $0-7$ & $8.34(6.08,11.6)$ & $8.47(6.59,10.6)$ & 0.316 \\
\hline Prealbumin (mg/L) & $200-430$ & $125(77,169)$ & $122(83,176)$ & 0.842 \\
\hline Albumin (g/L) & $40-55$ & $30.2(27.0,33.8)$ & $30.6(27.3,34.9)$ & 0.184 \\
\hline Bilirubin ( $\mu \mathrm{mol} / \mathrm{L})$ & $0-26$ & $12.0(9.28,17.2)$ & $11.8(9.15,15.3)$ & 0.316 \\
\hline Creatinine $(\mu \mathrm{mol} / \mathrm{L})$ & $57-97$ & $77.6(68.6,91.7)$ & $56.9(50.9,67.0)$ & $<0.001$ \\
\hline Creatine kinase (U/L) & $0-190$ & $96.0(62.3,174)$ & $58.0(38.5,95.0)$ & $<0.001$ \\
\hline CK-MB (U/L) & $0-24$ & $15.0(11.0,19.0)$ & $13.0(10.0,16.0)$ & $<0.001$ \\
\hline HS-CRP (mg/L) & $0-3$ & $55.8(19.3,115)$ & $25.3(5.28,60.9)$ & $<0.001$ \\
\hline Procalcitonin (ng/mL) & $0-0.05$ & $0.05(0.05,0.14)$ & $0.05(0.05,0.07)$ & $<0.001$ \\
\hline Troponin (pg/ml) & $0-28$ & $4.95(2.00,15.2)$ & $4.90(2.0,14.8)$ & 0.756 \\
\hline TC (mmol/L) & $3.3-5.2$ & $3.52(3.03,4.13)$ & $3.79(3.26,4.35)$ & 0.003 \\
\hline $\mathrm{HDL}(\mathrm{mmol} / \mathrm{L})$ & $1.29-1.55$ & $0.86(0.70,1.02)$ & $1.02(0.84,1.20)$ & $<0.001$ \\
\hline LDL (mmol/L) & $2.1-3.37$ & $2.06(1.62,2.55)$ & $2.05(1.61,2.55)$ & 0.849 \\
\hline TG (mmol/L) & $0.51-1.70$ & $1.69(1.49,2.06)$ & $1.77(1.55,2.17)$ & 0.051 \\
\hline ESR $(\mathrm{mm} / \mathrm{H})$ & $0-15$ & $47.4(32.8,63.0)$ & $51.5(38.5,67.4)$ & 0.110 \\
\hline
\end{tabular}

Data are median (IQR). $P<0.05$ was considered statistically significant. COVID-19, coronavirus disease 2019; ALT, alanine amino transferase; $L D H$, lactate dehydrogenase; IL-6, interleukin-6; CK-MB, creatine kinase isoenzyme-MB; HS-CRP, high sensitive C reaction protein; $T C$, total cholesterol; $H D L$, high-density lipoprotein; $L D L$, low-density lipoprotein; TG, triglyceride; ESR, erythrocyte sedimentation rate. Bold value means $P<$ 0.05 . decreased lymphocyte counts, platelet counts, total cholesterol (TC) and high-density lipoprotein (HDL). Compared to men, women have lower scrum high sensitive $c$ reaction protein (HSCRP) levels $(P<0.001)$. The sex-specific laboratory results of the 413 patients with COVID-19 were shown in Table 2.

\section{Clinical Outcomes}

The total of 31 patients (7.5\%) died during hospitalization. Figure 1 showed the sex-specific mortality in different age patients. The in-hospital mortality rate was $10.0 \%$ for men and $4.4 \%$ for women $(P=0.031)$. The cumulative survival rate was significantly different between males and females $(P=0.018$, log-rank test; Figure 2A). In the overall population, 91 cases (22.0\%) were diagnosed as severe condition. Although there was no significant difference in the severity of COVID-19 between males and females, severe cases were more likely to be seen in men than women $(24.3$ vs. $19.1 \%, P=0.203)$. There were no sex differences in development of ARDS, sepsis shock, acute kidney injury or acute liver injury (Table 3). However, compared with males, females were less likely to develop acute cardiac injury (6.6 vs. $13.5 \%, P=0.022$, Table 3 ).

\section{Multivariate Cox Regression of In-hospital Mortality}

Age and sex had significant interaction for the in-hospital mortality $(P<0.001)$ (Table 4$)$. Multivariate Cox regression analysis suggested increased in-hospital mortality was associated with age (HR 1.041, 95\% CI 1.009-1.073, $P=0.012$ ), male sex (HR 2.033, 95\% CI 1.007-2.098, $P=0.010$ ), the interaction between age and sex (HR 1.118, 95\% CI 1.003-1.232, $P=0.018)$, and comorbidities (HR 9.845, 95\% CI 2.280-42.520, $P=0.002$ ) (Table 4).

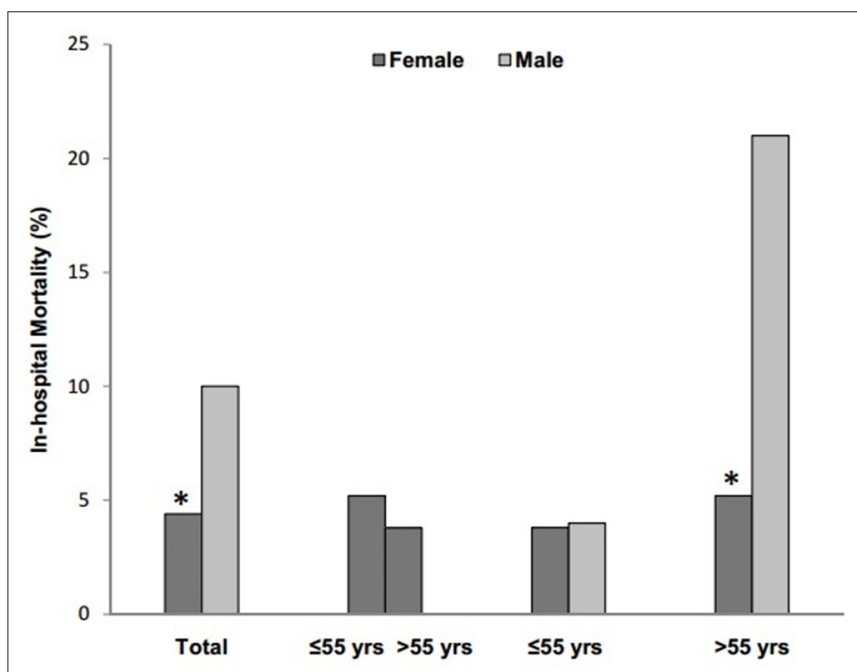

FIGURE 1 | Sex-specific in-hospital mortality of COVID-19 patients and subgroup stratified by age of 55 years. ${ }^{*} P<0.05$ vs. male by chi-squared tests or Fisher's exact tests. 

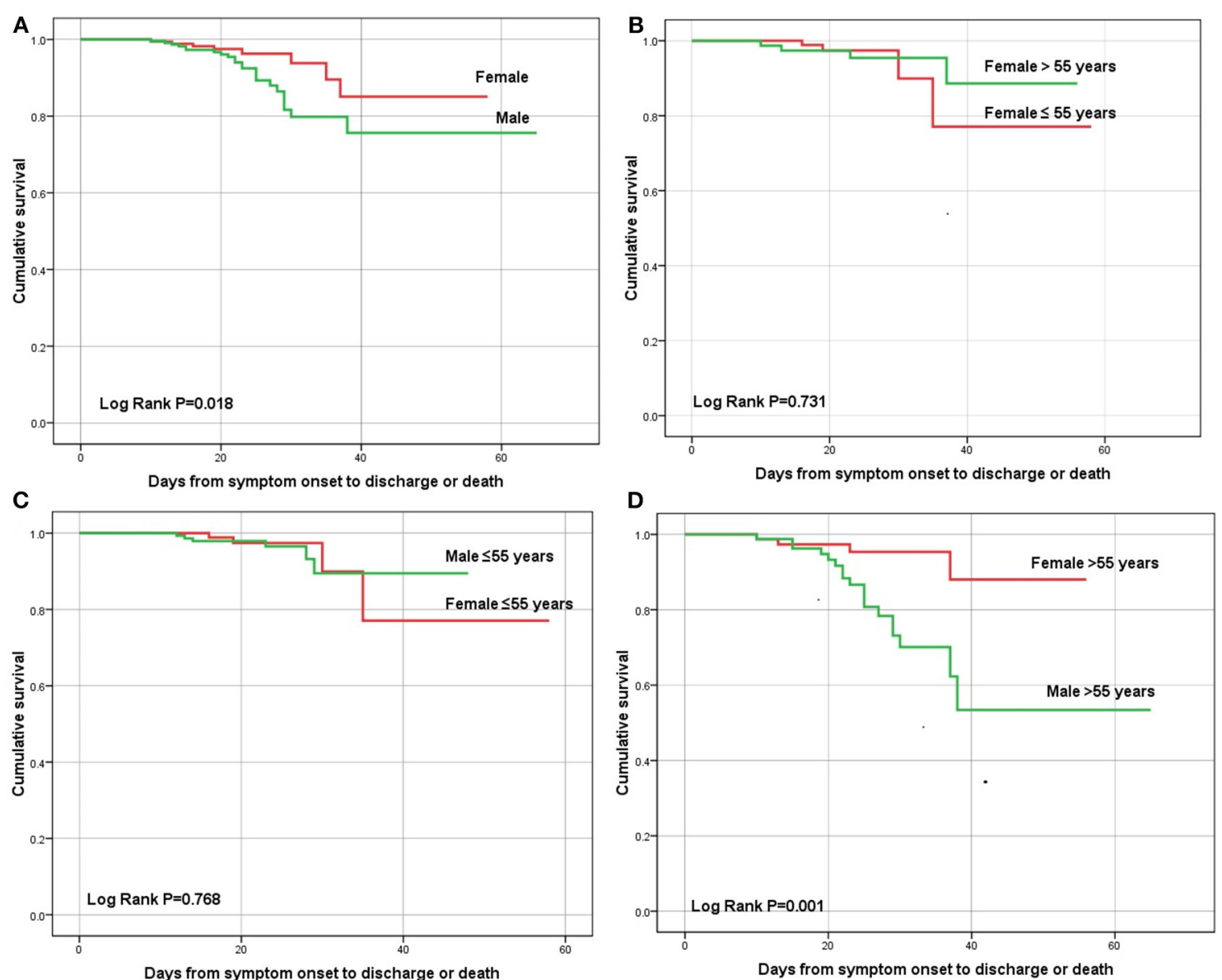

FIGURE 2 | Kalpan-Meier survival curve of male and female patients with COVID-19 in (A) male vs. female, (B) age $\leq 55$ years vs. age $>55$ years in female patients, (C) male vs. female in age $\leq 55$ years old group, and (D) male vs. female in age $>55$ years old group by log-rank test for all.

TABLE 3 | Sex-specific clinical outcomes of COVID-19 patients.

\begin{tabular}{|c|c|c|c|}
\hline Clinical outcomes & Male $(n=230)$ & Female $(n=183)$ & $P$-value \\
\hline Non-severe & $174(75.7)$ & $148(80.9)$ & - \\
\hline ARDS & $62(27.0)$ & $40(21.9)$ & 0.233 \\
\hline Time from symptom onset to ARDS, Median(IQR),d & $11(8,16)$ & $12(8,15)$ & 0.748 \\
\hline AKI & $18(7.8)$ & $10(5.5)$ & 0.343 \\
\hline Time from symptom onset to AKI, Median(IQR),d & $20(14,25)$ & $17(13,22)$ & 0.440 \\
\hline Acute liver injury & $33(14.3)$ & $18(9.8)$ & 0.166 \\
\hline Time from symptom onset to acute liver injury, Median(IQR),d & $17(11,23)$ & $15(11,19)$ & 0.840 \\
\hline Acute cardiac injury & $31(13.5)$ & $12(6.6)$ & 0.022 \\
\hline
\end{tabular}

Data are $n$ (\%) unless specified otherwise. $P<0.05$ was considered statistically significant. COVID-19, coronavirus disease 2019; ARDS, acute respiratory distress syndrome; IQR, inter quartile range; $A K I$, acute kidney injury. Bold value means $P<0.05$. 
TABLE 4 | Cox regression models evaluating risk factors associated with in-hospital mortality in COVID-19 patients.

\begin{tabular}{|c|c|c|c|c|c|c|}
\hline \multirow[t]{2}{*}{ Variables } & \multicolumn{2}{|c|}{ Univariate Cox regression } & \multicolumn{2}{|c|}{$\begin{array}{c}\text { Multivariate Cox regression } \\
\text { Model } 1\end{array}$} & \multicolumn{2}{|c|}{$\begin{array}{l}\text { Multivariate Cox regression } \\
\qquad \text { Model } 2\end{array}$} \\
\hline & HR (95\% Cl) & $P$-value & HR $(95 \% \mathrm{Cl})$ & $P$-value & HR (95\% Cl) & $P$-value \\
\hline Age & $1.054(1.023-1.087)$ & 0.001 & $1.056(1.026-1.085)$ & 0.001 & $1.041(1.009-1.073)$ & 0.012 \\
\hline Age $>55$ yrs (vs. Age $\leq 55$ yrs) & 3.755 (1.719-8.205) & 0.001 & & & & \\
\hline \multicolumn{7}{|l|}{ Sex } \\
\hline Male (vs. female) & $2.431(1.061-5.570)$ & 0.031 & $2.027(1.010-2.043)$ & 0.028 & $2.033(1.007-2.098)$ & 0.010 \\
\hline Age $\times$ Sex & $1.610(1.276-2.030)$ & $<0.001$ & $1.206(1.002-1.358)$ & 0.026 & $1.118(1.003-1.232)$ & 0.018 \\
\hline Comorbidities & $30.727(9.125-103.461)$ & $<0.001$ & & & $9.845(2.280-42.520)$ & 0.002 \\
\hline Median time from symptom onset to admission, $d$ & $0.672(0.543-1.384)$ & 0.396 & & & & \\
\hline Lymphocyte & $0.164(0.052-0.518)$ & 0.002 & & & & \\
\hline HS-CRP & 1.007 (0.992-1.023) & 0.353 & & & & \\
\hline Procalcitonin & $1.151(1.007-1.314)$ & 0.039 & & & & \\
\hline D-dimer & $1.016(0.991-1.040)$ & 0.210 & & & & \\
\hline Troponin & 1.002(0.996-1.009) & 0.487 & & & & \\
\hline TC & 0.889 (0.524-1.508) & 0.663 & & & & \\
\hline HDL & $1.356(0.365-5.041)$ & 0.649 & & & & \\
\hline SOFA & $1.881(1.546-2.289)$ & $<0.001$ & & & & \\
\hline
\end{tabular}

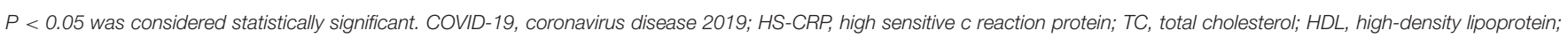

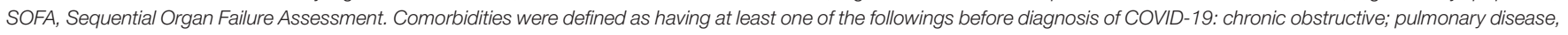

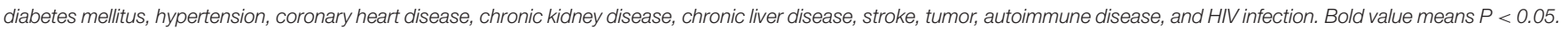

\section{Subgroup Analysis}

As mentioned earlier, female patients were divided into premenopausal and postmenopausal groups at the age of 55, and subgroup analysis was performed at the cut-off point of 55 years old. No differences on in-hospital mortality were noted in premenopausal women compared with postmenopausal women (3.8 vs. $5.2 \%, P=0.144$, Figure 1 ). In addition, subgroup analysis revealed no differences in-hospital mortality were noted in premenopausal women compared with men $(3.8$ vs. $4.0 \%, P=0.918)$, but differed significantly in postmenopausal women with men older than 55 (5.2 vs. $21.0 \%, P=0.007$ ). Additionally, no difference in cumulative survival rate was noted in premenopausal women compared with postmenopausal women $(P=0.731, \log$-rank test; Figure $2 \mathbf{B})$, as well as premenopausal women compared with men younger than $55(P=0.768, \log$-rank test; Figure 2C), but differed significantly in postmenopausal women with men older than $55(P=0.001$, log-rank test; Figure 2D).

In the subgroup analysis, acute liver injury and acute cardiac injury were less observed complications in women than men older than 55 (10.4 vs. $24.7 \%, P=0.019 ; 9.1$ vs. $25.9 \%, P=0.006$, respectively). However, the median duration from onset of symptoms to complications had no difference between sexes. Compared with male patients, female patients had higher lymphocyte $(P<0.001)$ and high-density lipoprotein $(P<0.001)$, lower high sensitive $c$ reaction protein level $(P<0.001)$, and lower incidence rate of acute cardiac injury (6.6 vs. $13.5 \%, P=0.022$ ). The sex differences of clinical characteristics and outcomes were shown in Tables 5-7.

\section{DISCUSSION}

To our best knowledge, this is the first multicentre retrospective cohort study to analyze the sex and estrogen effect on the clinical characteristics and outcomes in adult patients with SARS-COV-2 infection, and the role of estrogen in COVID-19 development. Estrogen modulates immune function in females and may contribute to resistance against infection, while estrogen level is significant difference before and after menopause. To explore the beneficial effect of estrogen in female COVID19 patients, we made the subgroup comparison according to the age of menopause in women. We documented that fever was more common in men cases, while digestive symptoms were less common. Men with COVID-19 were more prone to develop into the severe condition and die. The same trend was also found in Europe (31). Although no difference in-hospital mortality was noted in women under 55 compared with the same age men, there was significantly difference in women over 55 with men of the same age group, which may be associated with a lower inflammatory response in premenopausal women. However, differences in mortality between premenopausal and postmenopausal women was not significant, suggesting that female mortality in COVID-19 was lower than male might not be directly related to the effect of estrogen. Experimental data showed that male sex was an independent risk factor associated with refractory disease and death $(12,32,33)$. We also found that gender-related lifestyle, more chronic diseases, lower lymphocytes on admission, more complications and dyslipidemia may result in higher mortality rate in older men than in older women. These findings contribute to the discussion 
TABLE 5 | Sex-specific demographic and clinical characteristics for subgroup stratified by 55 years in COVID-19 patients.

\begin{tabular}{|c|c|c|c|c|c|c|}
\hline \multirow[t]{2}{*}{ Variables } & \multicolumn{3}{|c|}{ Age $\leq 55 Y(n=255)$} & \multicolumn{3}{|c|}{ Age $>55 Y(n=158)$} \\
\hline & Male $(n=149)$ & Female $(n=106)$ & $P$-value & Male $(n=81)$ & Female $(n=77)$ & $P$-value \\
\hline Age, Median(IQR), y & $47(37,51)$ & $46(38,50)$ & 0.494 & $66(61,71)$ & $66(62,73)$ & 0.280 \\
\hline current or ever smoking & $5(3.4)$ & $0(0)$ & 0.078 & $13(16.0)$ & $0(0)$ & $<0.001$ \\
\hline Drinking & $6(4.0)$ & $0(0)$ & 0.043 & $9(11.1)$ & $0(0)$ & 0.003 \\
\hline \multicolumn{7}{|l|}{ Comorbidities } \\
\hline COPD & $1(0.7)$ & $0(0)$ & NS & $5(6.2)$ & $1(1.3)$ & 0.236 \\
\hline $\mathrm{DM}$ & $7(4.7)$ & $4(3.8)$ & 0.720 & $23(28.4)$ & $20(26.0)$ & 0.733 \\
\hline Hypertension & $13(8.7)$ & $6(5.7)$ & 0.358 & $57(70.4)$ & $41(53.2)$ & 0.027 \\
\hline Heart disease & $3(2.0)$ & $2(1.9)$ & 0.943 & $12(14.8)$ & $9(11.7)$ & 0.563 \\
\hline Kidney disease & $1(0.7)$ & $1(0.9)$ & NS & $5(6.2)$ & $3(3.9)$ & 0.772 \\
\hline Liver disease & $2(1.3)$ & $2(1.9)$ & NS & $5(6.2)$ & 3 (3.9) & 0.772 \\
\hline Shock & $1(0.7)$ & $0(0)$ & NS & 7 (8.6) & $6(7.8)$ & 0.846 \\
\hline Tumor & $1(0.7)$ & $1(0.9)$ & NS & $5(6.2)$ & $6(7.8)$ & 0.689 \\
\hline Immune disease & $2(1.3)$ & $2(1.9)$ & NS & $2(2.5)$ & $2(2.6)$ & NS \\
\hline \multicolumn{7}{|l|}{ Symptoms } \\
\hline Fever & $141(94.6)$ & $86(81.1)$ & 0.001 & $67(82.7)$ & $64(83.1)$ & 0.947 \\
\hline Cough & $114(76.5)$ & $82(77.4)$ & 0.874 & 64 (79.0) & $60(77.9)$ & 0.868 \\
\hline Expectoration & $56(37.6)$ & $32(30.2)$ & 0.221 & 25 (30.9) & $25(32.5)$ & 0.829 \\
\hline Chest distress & $62(41.6)$ & 43 (40.6) & 0.867 & 48 (59.3) & 49 (63.6) & 0.572 \\
\hline Chest pain & $3(2.0)$ & $4(3.8)$ & 0.646 & $2(2.5)$ & $4(5.2)$ & 0.632 \\
\hline Hemoptysis & $2(1.3)$ & $1(0.9)$ & NS & $2(2.5)$ & $1(1.3)$ & NS \\
\hline Headache & $9(6.0)$ & 7 (6.6) & 0.855 & $3(3.7)$ & $4(5.2)$ & 0.945 \\
\hline Myalgia & $15(10.1)$ & $13(12.3)$ & 0.580 & $9(11.1)$ & $11(14.3)$ & 0.549 \\
\hline Fatigue & 53 (35.6) & 32 (30.2) & 0.369 & 30 (37.0) & 39 (50.6) & 0.085 \\
\hline Gastrointestinal & $19(12.8)$ & $17(16.0)$ & 0.458 & $14(17.3)$ & $18(23.4)$ & $<0.001$ \\
\hline SOFA Score, median (IQR) & $1(0,2)$ & $1(0,2)$ & 0.189 & $2(1,3)$ & $1(0,2)$ & 0.009 \\
\hline
\end{tabular}

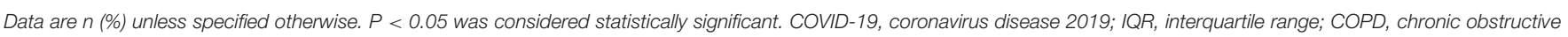
pulmonary disease; NS, no significance; DM, diabetes mellitus; SOFA, Sequential Organ Failure Assessment. Bold value means $P<0.05$.

of whether older male patients with SARS-COV-2 infection should be paid more attention.

It has been known that males and females differ in their susceptibility and response to viral infections, resulting in sex differences in incidence and disease severity $(18,34)$. The reduced susceptibility of females to viral infections could be attributed to the protection from $\mathrm{X}$ chromosome and sex hormones, which play an essential role in innate and adaptive immunity (35). SARS-CoV-2 uses ACE2 on pulmonary endothelium as an entry receptor, while the gene for the ACE2 is on the X chromosome (36), which may be the reason for the higher prevalence of COVID-19 in men than in women. In addition, in premenopausal women, estradiol produced by the ovary, is the estrogen in largest quantity $(40-400 \mathrm{pg} / \mathrm{mL})$ and most potent. Nevertheless, ovary almost stops producing estradiol after menopause, leading to estradiol level is significant reduced in postmenopausal women $(<20 \mathrm{pg} / \mathrm{mL})$, and no difference with men. In our study, the inflammatory marker HS-CRP was lowest in premenopausal women, while HS-CRP may result in cytokine storms and relate to disease severity and mortality $(2,6)$. Previous study showed hypopituitary women had decreased level of estrogen and increased level of CRP (37). Therefore, the lower morbidity and mortality of premenopausal women may be related to estrogen-mediated low inflammatory response. We also found that no difference in mortality among premenopausal and postmenopausal women. This suggested estrogen influenced the infection with SARS-COV-2 and pathogenesis of COVID-19, but might not be directly related to the lower mortality in women. However, our study had the small sample size, and the majority of younger patients are being non-severe COVID-19 condition and fewer died. Further investigation with larger -scale data is needed to assess the influence of estrogen on COVID-19 patients.

Although advancing age is associated with greater risk of death in both sexes, the male bias remains evident (15). Our study suggested that age, male sex and comorbidities were independently associated with in-hospital mortality, as well as sex and age had significant interaction for in-hospital mortality of COVID-19. The age-related sex differences in patients with COVID-19 were consistent with reported cases of seasonal and pandemic influenza A virus infections in Australia and Japan $(38,39)$. In the present study, we found the increasing mortality might be associated with higher rates of smoking, hypertension and complications in older men. Smoking rate is higher among men than women worldwide (40), consisting with the result 
TABLE 6 | Sex-specific laboratory results for subgroup stratified by 55 years in COVID-19 patients.

\begin{tabular}{|c|c|c|c|c|c|c|c|}
\hline Variables & Normal range & \multicolumn{3}{|c|}{ Age $\leq 55 Y(n=255)$} & \multicolumn{3}{|c|}{ Age $>55 Y(n=158)$} \\
\hline White blood cell $\left(\times 10^{9} / L\right)$ & $3.5-9.5$ & $7.77(6.77,10.2)$ & $7.45(6.55,9.10)$ & 0.341 & $7.98(6.76,11.1)$ & $7.76(6.71,9.51)$ & 0.346 \\
\hline Neutrophil $\left(\times 10^{9} / L\right)$ & $1.8-6.3$ & $5.93(4.95,7.90)$ & $5.89(4.65,8.03)$ & 0.713 & $6.27(4.87,8.45)$ & $6.00(4.68,8.60)$ & 0.464 \\
\hline Lymphocyte (×109/L) & $1.1-3.2$ & $1.35(1.07,1.77)$ & $1.45(1.13,1.74)$ & 0.532 & $1.13(0.84,1.40)$ & $1.31(1.10,1.67)$ & $<0.001$ \\
\hline Hemoglobin (g/L) & $316-354$ & $135(128,141)$ & $117(108,124)$ & $<0.001$ & $126(115,135)$ & $116(108,124)$ & $<0.001$ \\
\hline D-dimer $(\mu \mathrm{g} / \mathrm{ml})$ & $0-1.5$ & $0.56(0.31,1.15)$ & $0.62(0.33,1.17)$ & 0.720 & $1.11(0.68,4.15)$ & $1.25(0.58,3.85)$ & 0.803 \\
\hline ALT (U/L) & $9-50$ & $37.0(25.0,55.0)$ & $22.0(14.3,37.5)$ & $<0.001$ & $33.0(19.5,51.5)$ & $22.0(15.0,35.0)$ & $<0.001$ \\
\hline LDH (U/L) & $120-250$ & $331(284,397)$ & $327(281,404)$ & 0.941 & $356(290,479)$ & $338(278,429)$ & 0.041 \\
\hline IL-6 (pg/ml) & $0-7$ & $7.92(5.81,10.5)$ & $7.77(6.20,10.7)$ & 0.466 & $8.93(6.31,12.9)$ & $8.91(6.73,13.2)$ & 0.616 \\
\hline Prealbumin (mg/L) & $200-430$ & $151(117,215)$ & $137(92.5,194)$ & 0.079 & $89.0(56.8,129)$ & $109(79.0,155)$ & 0.003 \\
\hline Albumin (g/L) & $40-55$ & $30.0(26.8,33.7)$ & $30.9(27.0,35.4)$ & 0.107 & $30.3(27.0,33.8)$ & $30.4(27.4,33.9)$ & 0.795 \\
\hline CK-MB (U/L) & $0-24$ & $15.0(11.0,18.0)$ & $12.0(9.50,16.0)$ & 0.005 & $15.0(12.0,19.0)$ & $13.0(10.0,16.0)$ & 0.002 \\
\hline HS-CRP (mg/L) & $0-3$ & $26.7(11.88,82.4)$ & $12.9(3.90,39.3)$ & $<0.001$ & $93.6(44.3,131)$ & $26.0(10.1,63.9)$ & $<0.001$ \\
\hline Procalcitonin (ng/mL) & $0-0.05$ & $0.05(0.05,0.09)$ & $0.05(0.05,0.05)$ & $<0.001$ & $0.07(0.05,0.23)$ & $0.05(0.05,0.10)$ & 0.001 \\
\hline Troponin (pg/ml) & $0-28$ & $4.70(1.78,14.3)$ & $4.10(1.80,13.1)$ & 0.968 & $6.95(2.25,17.5)$ & $5.80(2.25,17.4)$ & 0.979 \\
\hline TC (mmol/L) & $3.3-5.2$ & $3.62(3.15,4.17)$ & $3.64(3.11,4.21)$ & 0.883 & $3.42(2.93,4.00)$ & $3.88(3.38,4.46)$ & $<0.001$ \\
\hline HDL (mmol/L) & $1.29-1.55$ & $0.85(0.71,1.00)$ & $1.00(0.81,1.19)$ & $<0.001$ & $0.87(0.70,1.04)$ & $1.05(0.85,1.21)$ & $<0.001$ \\
\hline LDL (mmol/L) & $2.1-3.37$ & $2.32(1.82,2.66)$ & $2.01(1.58,2.46)$ & 0.028 & $1.85(1.52,2.35)$ & $2.13(1.67,2.64)$ & 0.016 \\
\hline $\mathrm{TG}(\mathrm{mmol} / \mathrm{L})$ & $0.51-1.70$ & $1.73(1.54,2.15)$ & $1.78(1.59,2.19)$ & 0.444 & $1.65(1.45,1.99)$ & $1.77(1.52,2.15)$ & 0.024 \\
\hline ESR $(\mathrm{mm} / \mathrm{H})$ & $0-15$ & $46.7(35.3,61.5)$ & $49.5(29.2,61.9)$ & 0.725 & $53.5(28.1,63.0)$ & $58.6(43.5,72.2)$ & 0.065 \\
\hline
\end{tabular}

Data are median (IQR). $P<0.05$ was considered statistically significant. COVID-19, coronavirus disease 2019; ALT, alanine amino transferase; $L D H$, lactate dehydrogenase; IL-6, interleukin-6; CK-MB, creatine kinase isoenzyme-MB; HS-CRP, high sensitive c reaction protein; TC, total cholesterol; HDL, high-density lipoprotein; LDL, low-density lipoprotein; TG, triglyceride; ESR, erythrocyte sedimentation rate. Bold value means $P<0.05$.

TABLE 7 | Sex-specific clinical outcomes for subgroupstratified by 55 years in COVID-19 patients.

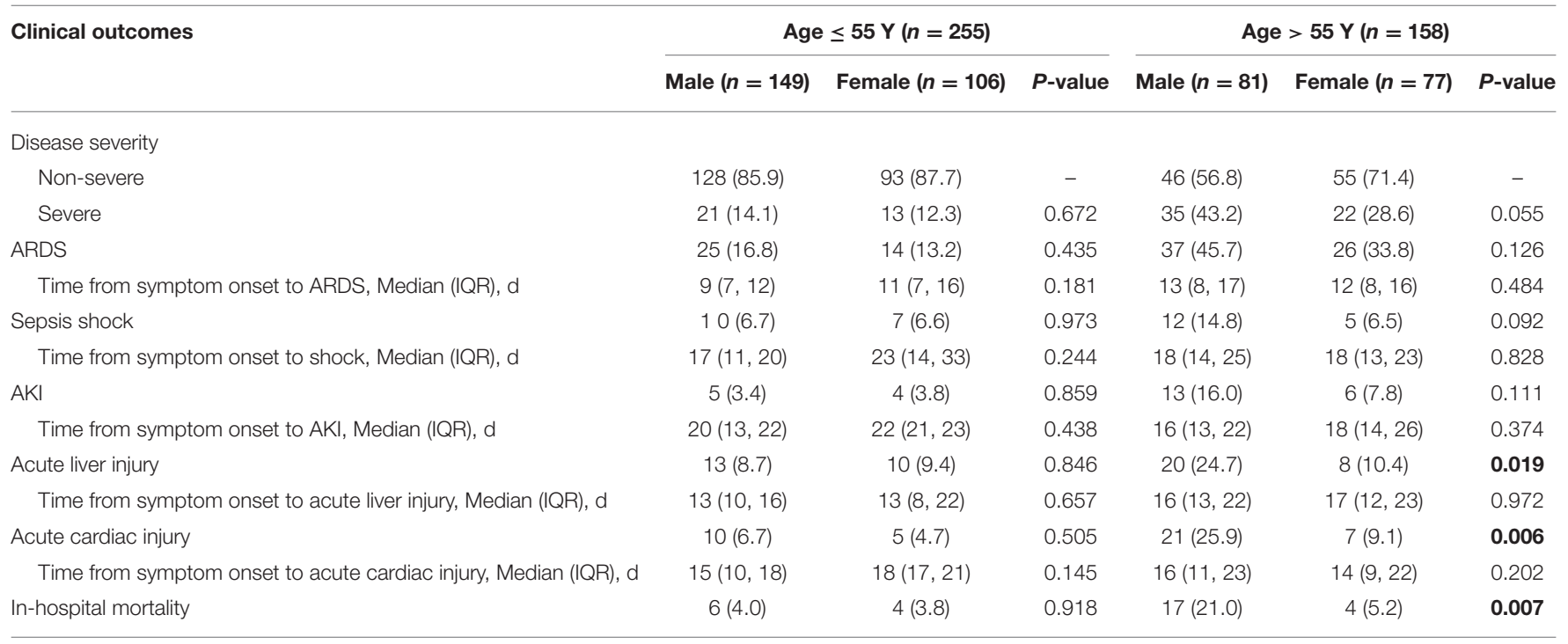

Data are $n$ (\%) unless specified otherwise. $P<0.05$ was considered statistically significant. COVID-19, coronavirus disease 2019; ARDS, acute respiratory distress syndrome; IQR, inter quartile range; AKI, acute kidney injury. Bold value means $P<0.05$. 
in this study. Such behavior is associated with the risk of developing comorbidities. Simultaneously, smoking is related to higher expression of ACE2 and may be a risk facto rfor disease prevalence and severity $(4,40,41)$, but no firm conclusions can be drawn. Hypertension was the most common chronic diseases, particularly in older male patients. Previous epidemiological data indicated an association between hypertension and severe disease or death from COVID-19 (7-9). Besides, the incidence of ARDS and other complications were higher in older men with COVID19 , especially acute liver injury and acute cardiac injury, which might also affect disease severity and prognosis.

In the older group, total lymphocyte count was significantly lower in men than in women. Lymphocytes play a decisive role in maintaining immune homeostasis and inflammatory response throughout the body. Previous studies had suggested that severe COVID-19 patients might have lymphocyte responses. A metaanalysis reported lymphopenia had a 3-fold higher risk of developing severe COVID-19, and lower lymphocyte counts was an effective biomarker in predicting the severity and prognosis in COVID-19 patients (42). Therefore, lymphopenia may be one potential mechanism of age-related sex differences.

In this study, dyslipidemia was found in older patients infected with SARS-COV-2. The TC, HDL, LDL, and TG levels in older male patients showed markedly decreases as compared to older female patients. This goes along well with previously data, that the LDL, HDL and TC levels in COVID-19 patients showed significant decreases at the time on admission (43). Lipids play a central role in viral infection, as they represent the structural foundations of cellular and viral membranes, while LDL levels inversely correlated to disease severities, which could be a predictor for disease progress and poor prognosis (43). The exact mechanisms of dyslipidemia in COVID-19 patients have remained unclear; however, there were several potential causes. Firstly, circulating lipid became oxidized in the inflammatory conditions, which resulted in the loss their protective functions and contributed to ongoing inflammation (44). Meanwhile, inflammation regulated lipoprotein metabolism indirectly via the cytokines, such as resulting in reduced expression and secretion of apoprotein of HDL, remodeling HDL-associated proteome, and promote HDL clearance from plasma $(45,46)$. Therefore, the measurement of the oxidized LDL and apoprotein of HDL can confirm these notions. Secondly, hepatocytes are the dominant cell type determining systemic LDL levels, and liver injury which is a common complication in older men, can affect the serum LDL levels. Thirdly, older male patients may have exacerbated inflammatory response, while systemic inflammation may accelerate clearance of LDL, which may partly explain the reduction in circulating LDL. Finally, estrogen levels in postmenopausal women affect the normal levels of blood lipids, which lead to increase TC, TG and LDL levels.

\section{LIMITATION}

There are several limitations in this study. Firstly, it was a retrospective multicentre study, and there were probably a significant referral bias, recall bias and measurement bias. Besides, this is an observational study and its results are subject to unobserved confounding factors. Secondly, we did not measure estrogen level and collect the history of hormone replacement therapy. However, we refer to previous studies and perform the subgroup analysis based on the age of menopause, to investigate the effect of estrogen in morbidity and mortality in patients with COVID-19 for the first time. Thirdly, only the indexes on admission were selected for analysis, without dynamic monitoring. Further, large-scale clinical studies and basic research are needed to explore risk factors for individualized assessment. Finally, no power calculation was made for the study, so the study is an exploratory study and its results are subject to false positive error and should be interpreted with caution.

In conclusion, older age, male sex and comorbidities were independently associated with in-hospital mortality of COVID19 patients. Moreover, sex and age are interactively associated with outcome of COVID-19. Although female mortality in COVID-19 is lower than male in this study, it might not be directly related to the effect of estrogen. Further study is warranted to identify the sex difference in COVID-19 and mechanisms involved.

\section{DATA AVAILABILITY STATEMENT}

The original contributions presented in the study are included in the article/supplementary material, further inquiries can be directed to the corresponding author/s.

\section{ETHICS STATEMENT}

The studies involving human participants were reviewed and approved by Jinan Infectious diseases Hospital, Shandong Provincial Chest Hospital, and Huanggang Central Hospital. The patients/participants provided their written informed consent to participate in this study.

\section{AUTHOR CONTRIBUTIONS}

YC and MM were involved in study concept and design and revised the final manuscript. JS, GQ, WS, CW, ZZ, XW, and $\mathrm{XB}$ collected the epidemiological and clinical data. PW, QY, and JJ processed statistical data. JS and GQ drafted the manuscript. All authors agree to be accountable for all aspects of the work in ensuring that questions related to the accuracy or integrity of any part of the work are appropriately investigated and resolved.

\section{FUNDING}

The work was supported by the National Natural Science Foundation of China (NSFC 81200238), and the Key Research and Development Projects of Shandong Province (2019GSF108059). 


\section{REFERENCES}

1. WHO Main Website. Available online at: https://www.who.int (accessed June 17, 2020).

2. Huang C, Wang Y, Li X, Ren L, Zhao J, Hu Y, et al. Clinical features of patients infected with 2019 novel coronavirus in Wuhan, China. Lancet. (2020) 395:497-506. doi: 10.1016/S0140-6736(20)30183-5

3. Yao Y, Tian Y, Zhou J, Ma X, Yang M, Wang S. Epidemiological characteristics of SARS-CoV-2 infections in Shaanxi, China by 8 February 2020. Eur Respir J. (2020) 55:2000310. doi: 10.1183/13993003.00310-2020

4. Guan WJ, Ni ZY, Hu Y, Liang WH, Ou CQ, He JX, et al. Clinical characteristics of coronavirus disease 2019 in China. N Engl J Med. (2020) 382:1708-20. doi: 10.1056/NEJMoa2002032

5. Guan W, Liang W, Zhao Y, Liang H, Chen Z, Li Y, et al. Comorbidity and its impact on 1590 patients with COVID-19 in China: a nationwide analysis. Eur Respir J. (2020) 55:2000547. doi: 10.1183/13993003.00547-2020

6. Ruan Q, Yang K, Wang W, Jiang L, Song J. Clinical predictors of mortality due to COVID-19 based on an analysis of data of 150 patients from Wuhan, China. Intensive Care Med. (2020) 46:846-8. doi: 10.1007/s00134-020-05991-x

7. Yang X, Yu Y, Xu J, Shu H, Xia J, Liu H, et al. Clinical course and outcomes of critically ill patients with SARS-CoV-2 pneumonia in Wuhan, China: a single-centered, retrospective, observational study. Lancet Respir Med. (2020) 8:475-81. doi: 10.1016/S2213-2600(20)30079-5

8. Wu C, Chen X, Cai Y, Xia J, Zhou X, Xu S, et al. Risk factors associated with acute respiratory distress syndrome and death in patients with coronavirus disease 2019 pneumonia in Wuhan, China. JAMA Intern Med. (2020) 180:934-43. doi: 10.1001/jamainternmed.2020.0994

9. Chow N, Fleming-Dutra K, Gierke R, Hall A, Hughes M, Pilishvili T, et al. Preliminary estimates of the prevalence of selected underlying health conditions among patients with coronavirus disease 2019 - United States, February 12-March 28, 2020. MMWR Morb Mortal Wkly Rep. (2020) 69:3826. doi: 10.15585/mmwr.mm6913e2

10. Luo H, Liu S, Wang Y, Phillips-Howard PA, Ju S, Yang Y, et al. Age differences in clinical features and outcomes in patients with COVID-19, Jiangsu, China: a retrospective, multicentre cohort study. BMJ Open. (2020) 10:e39887. doi: 10.1136/bmjopen-2020-039887

11. Di Castelnuovo A, Bonaccio M, Costanzo S, Gialluisi A, Antinori A, Berselli $\mathrm{N}$, et al. Common cardiovascular risk factors and in-hospital mortality in 3,894 patients with COVID-19: survival analysis and machine learning-based findings from the multicentre Italian CORIST study. Nutr Metab Cardiovasc Dis. (2020) 30:1899-913. doi: 10.1016/j.numecd.2020.07.031

12. Chen N, Zhou M, Dong X, Qu J, Gong F, Han Y, et al. Epidemiological and clinical characteristics of 99 cases of 2019 novel coronavirus pneumonia in Wuhan, China: a descriptive study. Lancet. (2020) 395:507-13. doi: 10.1016/S0140-6736(20)30211-7

13. Grasselli G, Zangrillo A, Zanella A, Antonelli M, Cabrini L, Castelli A, et al. Baseline characteristics and outcomes of 1591 patients infected with SARS-CoV-2 admitted to ICUs of the Lombardy Region, Italy. JAMA. (2020) 323:1574. doi: 10.1001/jama.2020.5394

14. Haitao T, Vermunt JV, Abeykoon J, Ghamrawi R, Gunaratne M, Jayachandran M, et al. COVID-19 and sex differences: mechanisms and biomarkers. Mayo Clin Proc. (2020) 95:2189-203. doi: 10.1016/j.mayocp.2020.07.024

15. Scully EP, Haverfield J, Ursin RL, Tannenbaum C, Klein SL. Considering how biological sex impacts immune responses and COVID-19 outcomes. Nat Rev Immunol. (2020) 20:442-7. doi: 10.1038/s41577-020-0348-8

16. Karlberg J, Chong DS, Lai WY. Do men have a higher case fatality rate of severe acute respiratory syndrome than women do? Am J Epidemiol. (2004) 159:229-31. doi: 10.1093/aje/kwh056

17. Alghamdi I, Hussain I, Alghamdi M, Almalki S, Alghamdi M, Elsheemy M. The pattern of Middle East respiratory syndrome coronavirus in Saudi Arabia: a descriptive epidemiological analysis of data from the Saudi Ministry of Health. Int J Gen Med. 7:417-23. doi: 10.2147/IJGM.S67061

18. Klein SL, Flanagan KL. Sex differences in immune responses. Nat Rev Immunol. (2016) 16:626-38. doi: 10.1038/nri.2016.90

19. Klein SL. The effects of hormones on sex differences in infection: from genes to behavior. Neurosci Biobehav R. (2000) 24:627-38. doi: 10.1016/S0149-7634(00)00027-0

20. Robinson DP, Huber SA, Moussawi M, Roberts B, Teuscher C, Watkins $\mathrm{R}$, et al. Sex chromosome complement contributes to sex differences in coxsackievirus B3 but not influenza A virus pathogenesis. Biol Sex Differ. (2011) 2:8. doi: 10.1186/2042-6410-2-8

21. Straub RH. The complex role of estrogens in inflammation. Endocr Rev. (2007) 28:521-74. doi: 10.1210/er.2007-0001

22. Channappanavar R, Fett C, Mack M, Ten Eyck PP, Meyerholz DK, Perlman S. Sex-based differences in susceptibility to severe acute respiratory syndrome coronavirus infection. J Immunol. (2017) 198:4046-53. doi: 10.4049/jimmunol.1601896

23. Interim Guidance for Novel Coronavirus Pneumonia (Trial Implementation of Revised Fifth Edition). National Health and Health Commission (2020). Available online at: http://www.nhc.gov.cn/yzygj/s7653p/202002/ d4b895337e19445f8d728fcaf1e3e13a.shtml (accessed May 8, 2020).

24. Ning NG, Yun WX, Yan YH. The research on the factors affecting the timing of natural menopause in Chinese city women. Maternal Child Health Care China. (2011) 26:1191-3.

25. Davis M, Diamond J, Montgomery D, Krishnan S, Eagle K, Jackson E. Acute coronary syndrome in young women under 55 years of age: clinical characteristics, treatment, and outcomes. Clin Res Cardiol. (2015) 104:64855. doi: 10.1007/s00392-015-0827-2

26. Metlay JP, Waterer GW, Long AC, Anzueto A, Brozek J, Crothers K, et al. Diagnosis and treatment of adults with community-acquired pneumonia. An official clinical practice guideline of the American Thoracic Society and Infectious Diseases Society of America. Am J Respir Crit Care Med. (2019) 200:e45-67. doi: 10.1164/rccm.201908-1581ST

27. Singer M, Deutschman CS, Seymour CW, Shankar-Hari M, Annane D, Bauer $\mathrm{M}$, et al. The third international consensus definitions for sepsis and septic shock (sepsis-3). JAMA. (2016) 315:801-10. doi: 10.1001/jama.2016.0287

28. Khwaja A. KDIGO clinical practice guidelines for acute kidney injury. Nephron Clin Pract. (2012) 120:c179-84. doi: 10.1159/000339789

29. Ranieri VM, Rubenfeld GD, Thompson BT, Ferguson ND, Caldwell E, Fan E, et al. Acute respiratory distress syndrome: the Berlin Definition. JAMA. (2012) 307:2526-33. doi: 10.1001/jama.2012.5669

30. Zhou F, Yu T, Du R, Fan G, Liu Y, Liu Z, et al. Clinical course and risk factors for mortality of adult inpatients with COVID-19 in Wuhan, China: a retrospective cohort study. Lancet. (2020) 395:105462. doi: 10.1016/S0140-6736(20)30566-3

31. Gebhard C, Regitz-Zagrosek V, Neuhauser HK, Morgan R, Klein SL. Impact of sex and gender on COVID-19 outcomes in Europe. Biol Sex Differ. (2020) 11:29. doi: 10.1186/s13293-020-00304-9

32. Mo P, Xing Y, Xiao Y, Deng L, Zhao Q, Wang H, et al. Clinical characteristics of refractory COVID-19 pneumonia in Wuhan, China. Clin Infect Dis. (2020) 16:ciaa270. doi: 10.1093/cid/ciaa270

33. Zhang J, Wang X, Jia X, Li J, Hu K, Chen G, et al. Risk factors for disease severity, unimprovement, and mortality in COVID-19 patients in Wuhan, China. Clin Microbiol Infect. (2020) 26:767-72. doi: 10.1016/j.cmi.2020.04.012

34. Markle JG, Fish EN. SeXX matters in immunity. Trends Immunol. (2014) 35:97-104. doi: 10.1016/j.it.2013.10.006

35. Jaillon S, Berthenet K, Garlanda C. Sexual dimorphism in innate immunity. Clin Rev Allerg Immu. (2019) 56:308-21. doi: 10.1007/s12016-017-8648-x

36. Patel SK, Velkoska E, Freeman M, Wai B, Lancefield TF, Burrell LM. From gene to protein-experimental and clinical studies of ACE2 in blood pressure control and arterial hypertension. Front Physiol. (2014) 5:227. doi: 10.3389/fphys.2014.00227

37. Sesmilo G, Miller KK, Hayden D, Klibanski A. Inflammatory cardiovascular risk markers in women with hypopituitarism. J Clin Endocrinol Metab. (2001) 86:5774-81. doi: 10.1210/jcem.86.12.8087

38. Eshima N, Tokumaru O, Hara S, Bacal K, Korematsu S, Tabata M, et al. Sex- and age-related differences in morbidity rates of 2009 pandemic influenza A H1N1 virus of swine origin in Japan. PLoS ONE. (2011) 6:e19409. doi: 10.1371/journal.pone.0019409

39. Wong KC, Luscombe GM, Hawke C. Influenza infections in Australia 2009-2015: is there a combined effect of age and sex on susceptibility to virus subtypes? BMC Infect Dis. (2019) 19:42. doi: 10.1186/s12879-0193681-4

40. Cai H. Sex difference and smoking predisposition in patients with COVID-19. Lancet Respir Med. (2020) 8:e20. doi: 10.1016/S2213-2600(20)30117-X

41. Walter LA, McGregor AJ. Sex- and gender-specific observations and implications for COVID-19. West J Emerg Med. (2020) 21:507-9. doi: 10.5811/westjem.2020.4.47536 
42. Zhao Q, Meng M, Kumar R, Wu Y, Huang J, Deng Y, et al. Lymphopenia is associated with severe coronavirus disease 2019 (COVID-19) infections: a systemic review and meta-analysis. Int J Infect Dis. (2020) 96:1315. doi: 10.1016/j.ijid.2020.04.086

43. Fan J, Wang $\mathrm{H}, \mathrm{Ye} \mathrm{G}, \mathrm{Cao} \mathrm{X}, \mathrm{Xu} \mathrm{X}$, Tan $\mathrm{W}$, et al. Letter to the editor: low-density lipoprotein is a potential predictor of poor prognosis in patients with coronavirus disease 2019. Metab Clin Exp. (2020) 107:154243. doi: 10.1016/j.metabol.2020.154243

44. Guirgis FW, Dodani S, Moldawer L, Leeuwenburgh C, Bowman J, Kalynych C, et al. Exploring the predictive ability of dysfunctional high-density lipoprotein for adverse outcomes in emergency department patients with sepsis. Shock. (2017) 48:539-44. doi: 10.1097/SHK.0000000000 000887

45. van der Westhuyzen DR, de Beer FC, Webb NR. HDL cholesterol transport during inflammation. Curr Opin Lipidol. (2007) 18:147-51. doi: 10.1097/MOL.0b013e328051b4fe
46. McGillicuddy FC, de la Llera Moya M, Hinkle CC, Joshi MR, Chiquoine EH, Billheimer JT, et al. Inflammation impairs reverse cholesterol transport in vivo. Circulation. (2009) 119:113545. doi: 10.1161/CIRCULATIONAHA.108.810721

Conflict of Interest: The authors declare that the research was conducted in the absence of any commercial or financial relationships that could be construed as a potential conflict of interest.

Copyright $\odot 2021$ Sha, Qie, Yao, Sun, Wang, Zhang, Wang, Wang, Jiang, Bai, Chu and Meng. This is an open-access article distributed under the terms of the Creative Commons Attribution License (CC BY). The use, distribution or reproduction in other forums is permitted, provided the original author(s) and the copyright owner(s) are credited and that the original publication in this journal is cited, in accordance with accepted academic practice. No use, distribution or reproduction is permitted which does not comply with these terms. 